\title{
ON THE ANALYSIS OF BUTTER.
}

By Dr. JoHn Muter, F.C.S.

Read at an Ordinary Meeting of the Society of Public Analysts, held March 15th, 1876.

Butrer differs from all other fats inasmuch as it contains a notable proportion of fatty acids other than oleic, stearic, palmitic, and their congeners. If we analyse the pure glycerides of these latter acids we obtain, by the taking up of three molecules of water during saponification and subsequent liberation of the acids, amounts which almost exactly approximate themselves to theory. There is no process in the whole range of analytical chemistry more accurate in the hands of those who know the importance of never trusting to the eye to decide whether ressels, \&c., are free from fat, but of invariably drying every article used, and extracting it afterwards with ether. If precision is to be attained, the principles of mineral analysis must not be applied. Fat precipitates cannot be treated as if they were barium sulphate, and expense in the shape of such articles as ether and absolute alcohol must not be spared. I am entitled to put this point strougly before public analysts, because of the experience in fats possessed by myself and my assistants owing to our having been specially thrown into this branch of analysis some years ago. Unfortunately, with a laudable desire no doubt to introduce simplified processes, Messrs. Angell \& Hehner have touched dangerous ground, and proposed the washing of fatty acids on a filter, with hot water, taking it for granted that because no fat was visibly coming through, they could manipulate thus in safety. I will venture to say, however, that every one of the few chemists who have been called upon to work in fats for commercial or scientific purposes, would agree with me that such a process is in the highest degree dangerous. Not only should fatty acids never be trusted out of the vessel in which they are precipitated until they are finally transferred to the weighing capsule, but also every rod, beaker, and even the filter paper used to pass the washings, should be dried and extracted with a suitable solvent. Mr. Angell, himself, evidently feels the difficulty, because in his book he gives a special instruction that "great care must be observed in the washing." I hold that processes requiring such. delicate care are not suitable for use under a penal statute; and, indeed, I go further and saj, that with even the highest precaution, a constant loss of at least 5 centigrams to 1 decigram is made in every analysis on this principle, using 5 grams of fat. By accurate methods, such as it will be my aim to point out in this paper, the following pure glycerides can be so nearly analysed to theory as to be made to show :-

\begin{tabular}{|c|c|c|}
\hline Tristearin & 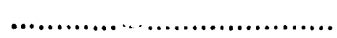 & $95 \cdot 70$ \\
\hline Triolein & 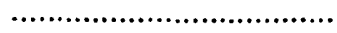 & $95 \cdot 52$ \\
\hline 'Tripalmatin & & $95 \cdot 27$ \\
\hline
\end{tabular}

and no process of washing on a filter ever could come witlin, at the nearest, half a per cent. of these results, except by an accident. A very striking case in point is afforded by Messrs. Angell \& Hehner. After giving analyses of tallow, lard, and cocoa butter (the latter two being, by the way, about 5 per cent. short of the ordinary yield), they introduce an analysis of palm oil, in which they admit a deficiency, but fancy that it is accounted for by colouring matter. Now palm oil is a specialty of ours, one of my staff having for six months done nothing else almosí, and I can assure you that there is no palm oil in the market jielding so low an amount of fatty acids by at least 2 per cent. Indeed it is a fact that commercial palm oil always yields over theory owing to free acidity, and the most highly coloured samples give no appreciable loss of weight when 
the colour is destroyed by heat. Here, therefore, is the decigram loss in 5 grams plainly manifest.

Besides the glycerides already referred to, butter contains tributyrin in considerable proportion, with small traces of tricaproin and tricaprylin. I have not yet sufficiently separated these traces for estimation, as their fractional liberation from barium is very troublesome; but of this much I am certain, that they are only present to a very small amount. That this is so may be proved by supposing a butter to yield 88 per cent. of insoluble acids (oleic, stearic, and palmitic, the latter two being present in the proportion of the so-called Margaric acid of older writers). This will represent $92 \cdot 14$ per cent. of glycerides, leaving $7 \cdot 86$ for glycerides of the soluble acids. If the latter were all tributyrin it would give a soluble acidity of 6.88 , but in practice this does not come out. Taking a butter yielding an amount nearly like 88, we have-

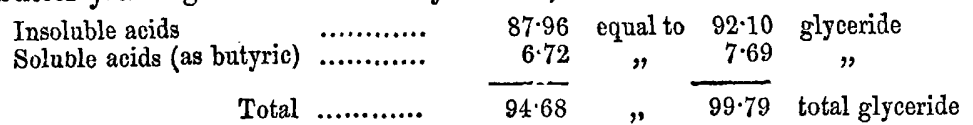

thus showing a deficiency of about 2 per cent. owing to the traces of higher soluble acids. The difference thus shown may be disregarded, as, although a little variable, it always comes within $\cdot 7$ per cent. Calculating therefore always as butyric, we come to this factthat no analysis of butter can be held to be complete unless both the soluble and insoluble acids be estimated, and they come, when added together, within a fair range of $94 \cdot 8$, allowing for possible experimental errors to, say, the extent of $\cdot 3$ to $\cdot 5$ per cent. in either direction.

The process $I$ adopt for the full analysis of butter is as follows : -

(1) 1500 grains of the butter are placed in a counterpoised porcelain dish, over a very low gas flame, and stirred with a thermometer at a heat not exceeding $230 \mathrm{deg}$. F. until all the water is driven off, which is indicated by effervescence entirely ceasing, and the curd and salt settling perfectly down to the bottom of the dish, leaving the absolutely clear melted fat. The whole is then cooled and weighed, and the loss calculated to percentage of water. This is the only method of absolutely and rapidly drying a fat, and the large quantity taken ensures a more perfect estimate of the true amount of water in the sample. I have proved by careful experiment that the temperature of $230 \mathrm{deg}$. has not the slightest influence on butter fat.

(2) The fat is melted at a gentle heat, and poured off as far as possible into a beaker, without disturbing the sediment. The remainder is poured on a weighed filter, placed over a beaker in the drying chamber, and, when all is through, the basin and filter are rinsed with petroleum spirit to remove all the traces of fat, and the filter being dried and weighed gives curd plus ash.

(3) The filter after being weighed is placed in a weighed platinum crucible, and gently ignited. This gives ash, called salt in the report.

(4) The fat poured off from (2)-which will generally be about 1200 grains-if absolutely cleur, is at once used for physical and chemical examination; but if not perfectly free from specks it must be filtered through a Swedish filter kept hot on the water bath. The processes necessary are, the taking of the specific gravity of the fat at $100 \mathrm{deg}$. F., and if that gives an adverse indication, the estimation of the total fatty acids of the butter fat both soluble and insoluble. 
FIRst.-The "Actual Density" at $100 \mathrm{deg}$. F.-This process was first publicly described by Mr. Bell, of the Inland Revenue Laboratory, in the Southwark Police Court. As employed by him, however, the results do not appear to be those of actual density, nor do I consider that the precautions to ensure accuracy are quite sufficient, considering the rapid expansibility of melted fats by heat. I will give his process in his own words. "The fat is taken out of the water bath and poured into the bottle until it is filled up to " the neck. One person then takes the bottle and another the residue of the fat, and " both are brought to exactly $100 \mathrm{deg}$. F., when the bottle is filled from the residue and " stoppered in the usual way." Now I have tried this method but I find that, supposing the fat to be taken from the bath at, say, $200 \mathrm{deg}$., and each person cools his portion to 100 , then the pouring in and stoppering will frequently, by a little want of care, cause the bottle to be closed when a part of its contents has gone below the 100 , to the extent of 2 degrees; because when fat is taken at 100 "on the fall," it will lose a degree of heat almost in a few seconds. At all events, the process can never be absolutely certain within one or two grains on the 1000 grain bottle. The results he gave in court embrace a range from 909.00 to 905.00 , and these at once show that the actual density is not indicated.

I take the actual density of a fluid to be the weight of any given volume of it, as compared with that of an equal bulk of distilled water at the same lemperature. These results compare butter at $100 \mathrm{deg}$. with water at $60 \mathrm{deg}$. to $62 \mathrm{deg}$. F., and are not therefore actual densities; and I submit, that to get the true advantage of inequality of expansion, the water and butter must both be taken at $100 \mathrm{deg}$. F. The process I adopt is as follows :-

A 1000 grain bottle is procured with rather a pear-shaped neck, and fitted with a thermometer stopper ranging from $32 \mathrm{deg}$. to $140 \mathrm{deg}$. F. The long mercurial bulb comes exactly down the centre of the bottle, and the scale is up above the stopper. The bottle is placed on the balance, and an accurate counterpoise prepared for it. It is then filled with recently boiled distilled water, at $95 \mathrm{deg}$. F. The stopper is inserted, and the whole at once plunged up to the neck into a $12 \mathrm{oz}$. squat beaker partially filled with distilled water at $103 \mathrm{deg}$. F. in which is placed a thermometer. As the temperature rises in the bottle, the water leaks out at the stopper, and in a few minutes (if the quantity of water in the beaker be properly regulated), a time arrives when the temperature of both thermometers equalise themselves at $100 \mathrm{deg}$. The joint between the stopper and the bottle is instartly wiped with a small piece of filter paper to absorb loose water, and the bottle is lifted out, thoroughly cleansed and weighed. By repeating this three times the actual contents of the bottle at $100 \mathrm{deg}$. F. is obtained, and the weight taken, before a fall of more than 5 degrees takes place. At first I let the bottle cool to 60 degrees so as to avoid currents; but $\mathbf{I}$ found that it was better in practice to weigh at once, and quite as accurate. This weight of water is scratched on the bottle with a diamond, and all is ready for the butter. The pure butter fat, prepared as already described, is taken from the bath and cooled to $95 \mathrm{deg}$. F., it is then poured into the bottle, and the whole operarion repeated thrice, exactly as with the water, and the mean of the three weighings thus obtained is divided by that of the water. The contrivance of having a "rising" fat heated by a "falling" water until the two equalize, is the height of accuracy, and moreover gives an appreciable rest in the rariation of the temperature sufficient to enable the excess of fat which has leaked out to be removed exactly at the required temperature. 
Mr. Bell stated in court that there was an analogy between the specific gravity and the per centage of insoluble fatty acids, and here he is correct. The following are som of the figures he has given compared with the true results found by full analyses, the worst of which came to within $\cdot 5$ of the truth on the whole addition :-

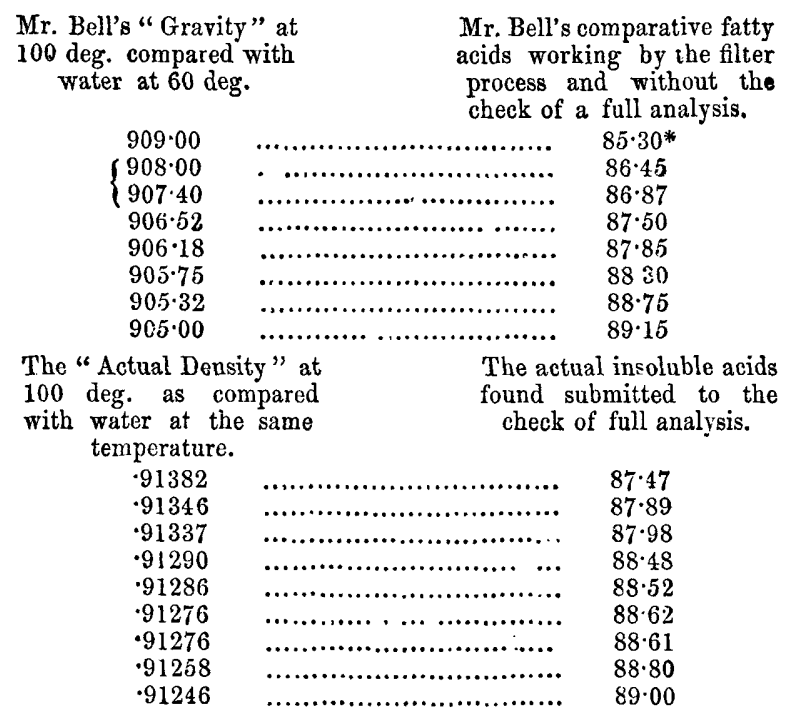

The whole nine examples by my process are corresponding to butters between 908 and 907 by Mr. Bell's gravity, and the results show a much higher and more regular per centage of fatty acids. The regular loss on the filter process I have already referred to, is strikingly manifested by the following comparison. I happened to have a butter which gave 91286 actual density, corresponding exactly to 907:4 of Mr. Bell's gravity, and so we see by the filter washing Mr. Bell makes that show 86.87 , while on fall analysis by my process it shows 88.52 , or as nearly as possible the decigram in five grams difference, as seen in the palm oil experiment.

While therefore we must admit the great correspondence of the density and acids when both are properly taken, it is to be noted that the moment you come to mix butter with other fats the whole thing is upset for quantitative purposes. The fats used for butter mixing are sume of them of an "actual density" of .90659 (dripping) to 90294 (vegetable "butterine,") and therefore all we can say as regards specific gravity is that if a butter shows anything over 91100 "actual density," it may safely be passed over without further analysis as being good.

Second.-7he Total Fatty Acids.-About 10 grams (or 150 grains) of the butter fat at $100 \mathrm{deg}$. F. are weighed by difference from a suspended tube into a clean dry 15 ounce flask, and 5 grains of Potassium Hydrate, with 2 fluid ounces of rectified spirit are added. The flask is placed in a basin with hot water, and kept boiling for a considerable time, until on adding water not the faintest turbidity occurs. Ten ounces of water are added, and evaporation continued (just short of boiling) until all traces of Alcohol are dissipated. The contents of the flask are then made up to 7 ounces, with nearly boiling water, and a good fitting cork having been introduced, through which just passes a tube 2 feet long and ending in a small funnel, 5 grammes of full strength

* This result is an extraordinary instance of the filter process. No such butter is to be found in nature. 
Sulphuric Acid are poured in down the tube followed by some water. The whole is then agitated with a circular motion until the soap, which rises suddenly, is changed into a perfectly clear and transparent stratum of fatty acids. The flask and contents are then cooled down to $40 \mathrm{dg}$. F., till a perfectly solid cake of fatty acid forms. A few drops of cold water are run in to wash the tube, and the cork having been removed, a small piece of fine cambric is placed over the mouth of the flask, held in situ by an ordinary India rubber ring. The fat cake is caused to detach itself from the sides of the flask by a gentle movement, and then the filtrate is decanted, without breaking the cake, into a litre test mixer, with a good stopper. About an ounce of cold water is poured into the flask through the cambric, and the whole cake and Hask rinsed out by gently turning round and the washings added to the filtrate. Six ounces of water at 120 deg. are now added through the muslin, which is then quickly detached, and the cork and tube inserted. The whole is again heated, this time to $200 \mathrm{deg}$., and kept constantly agitated with a circular, but not a jerky motion for five minutes. This agitation so divides the fat, that it almost forms an emulsion with the water, and is the only means of thoroughly and rapidly washing fatty acids without loss. In practice no Butyric Acid comes off at 200 deg., but any trace that might do so, is caught in the long tube. The cooling and filtering are then again proceeded with as above deseribed, (the filtrate being added to the contents of the test mixer,) and the washings are repeated alternately cold with 1 ounce, and hot with 6 ounces of water, until they do not give the slightest change to neutral litmus. After thoroughly draining the residual cake by letting the flasks stand upside down for some time, the cambric is removed and the flask is laid out on its side in the drying oven, with a support under the neck, until the acids are thoroughly fused, when they are poured while hot into a tared platinum capsule, dried and weighed. The film of fatty acid still remaining on the flask is rinsed out with ether, and dried in a small weighed beaker, and the weight added to the whole. If any drops of water be observed under the fatty acids in the capsule after an hour's drying, the addition of a few drops of absolute alcohol will quickly cause them to dry off. If any trace of fat is on the cambric it should be also dried and extracted with ether, but with eare not to break the cake at the last pouring off, this does not occur.

The process is absolutely accurate, and the merest tyro cannot make any loss so long as he does not deliberately shake the melted acids against the cork, which he could not do if he practices a circular agitation while washing.

The filtrate in the test mixer is now made to an absolute bulk, and in $200 \mathrm{c:c}$ : the total acidity is taken with a weak solution of sodium hydrate. The solution $I$ generally use represents $\cdot 01$ of $\mathrm{N} \mathrm{H}_{3}$ in each c.c., as it serves also for nitrogen combustions ; but a useful strength would be decinormal soảa, containing $\cdot 004 \mathrm{Na} \mathrm{H} \mathrm{O}$ in each c.c: The acidity found is multiplied by five, calculated to $\mathrm{H}_{2} \mathrm{SO}_{4}$ and noted as "total acidity as $\mathrm{H}_{2} \mathrm{SO}_{4}^{\prime \prime}: 100$ c.c. are next taken, and presipitated with barium chloride in the presence of a strong acidulation, with hydrochloric acid, well boiled and washed by three decantations, boiling each time; and, lastly, on a filter, till every trace of soluble barium is removed. The precipitate is dried, ignited, and weighed as usual, multiplied by ten, and calculated to $\mathrm{H}_{2} \mathrm{SO}_{4}$, and noted as "total sulphuric acid." Lastly, 100 c.c. are evaporated to dryness over the water bath in a tared platinum dish holding 120 c.c., and furnished with a cover of platinum foil, also tared. When dry the dish is covered and heated over a bunsen till all fumes cease, and a fragment of pure ammonium carbonate 
having been added, the whole is again ignited and weighed. The amount of potassium sulphate found is multiplied by 10 and calculated to $\mathrm{H}_{2} \mathrm{SO}_{4}$, and noted as "combined sulphuric acid." The rest of the calculation is obvious to any analyst, but $I$ give an example :

Ten grammes taken.

$$
\begin{aligned}
& \text { Total acidity as } \mathrm{H}_{2} \mathrm{SO}_{4} \quad \ldots \ldots \ldots \ldots \ldots . . . .6 .814 \\
& \text { Total } \mathrm{H}_{2} \mathrm{SO}_{4} \quad \text {............... } 4.9 \\
& \text { Combined } \mathrm{H} \mathrm{SO}_{4} \quad \text {............... } 4.4 \\
& 4.9-4.4=.5 \text { free } \mathrm{H}_{2} \mathrm{SO}_{4} \\
& 0 \cdot 814-5=-314 \text { acidity due to butter acids stated as } \mathrm{H}_{2} \mathrm{SO}_{4} \\
& \text { Then } \frac{.314 \times 176}{98}=\cdot 564 \text { butyric acid in } 10 \text { grammes taken which equals } 5.64 \text { per cent. }
\end{aligned}
$$

By this means we get the soluble acids indirectly by processes which are the every day work of nearly all commercial analysts.

I have only to remark that the barium sulphate should always be washed very carefully, and seeing that it regularly weighs a little over a gramme, it is advisable to boil up with dilute hydrochloric acid after ignition and see that clear liquid gives no cloud with sulphuric acid.

The following may be taken as fair specimens selected from a great mass of results by my process, and as a proof of the almost impossibility of error we have the check given by the totals found. I may also state, that another fact which speaks well is, that I have taken the same sample, one to two months apart, without getting one-tenth per cent. variation in the amount of insoluble fatty acids

I.-A rich butter which by theory from the density should yield

$$
\begin{array}{lrr}
\text { Soluble acids } & \ldots \ldots \ldots \ldots \ldots . . & 7 \cdot 05 \\
\text { Insoluble acids } & \ldots \ldots \ldots \ldots . . . & 87 \cdot 8 \\
& & 94.85
\end{array}
$$

was analysed twice and in cach analysis two determinations of sulphates were made.

Soluble acids

Insoluble acids

$$
\begin{array}{ccccccr}
1 \text { st } & \text { Analysis. } & \multicolumn{4}{c}{2 \text { 2nd Analysis. }} \\
6.92 & \ldots & 6 \cdot 89 & \ldots \ldots . . & 6 \cdot 85 & \ldots & 6.93 \\
\frac{87 \cdot 86}{94 \cdot 78} & \cdots & \frac{87 \cdot 86}{94 \cdot 75} & \ldots \ldots . . & \frac{87 \cdot 87}{94 \cdot 72} & \ldots & \frac{87 \cdot 87}{94 \cdot 80}
\end{array}
$$

II. - A poor butter showing by theory from density 88.8 -

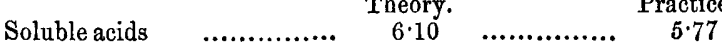

$$
\begin{aligned}
& \text { Insoluble acids } \quad \text {.............. } \frac{88 \cdot 8}{94 \cdot 90} \quad \cdots \cdots \cdots \cdots \cdots \cdots \quad \frac{88.95}{94 \cdot 72}
\end{aligned}
$$

III.-A butter showing from density 89

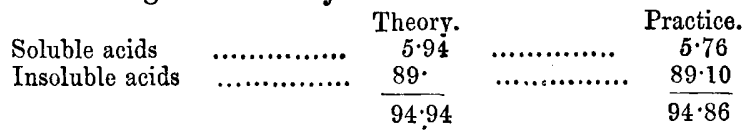

IV.-A sample of butter purchased at the same shop and same price as the Southwark disputed butter, and showing a similar amount of insoluble acid-

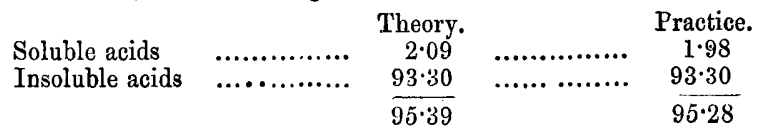

V.-A sample of Belgian "Butterine," from vegetable fat-

Soluble acids Theory. 
These are only a few of the mass of results I have, which I have selected to show my views of the composition of butter. They are each examples of a special class. No. I. being a first-class Aylesbury butter. No II. an old low-class Dutch butter, kept six months, until quite unfit for food. No. III. a butter which had lost all character, and was not distinguishable from a piece of tallow, although genuine Friesland eight months ago. No. IV. was evidently two-thirds foreign fat; and No. V. was all vegetable fat, and the two-tenths per cent. of soluble acids are an experimental error.

I intend, if able to spare the time, to return to the subject at our next meeting, and answer the questions (1) What is the average composition of natural butter? and (2) How far may the butter be affected by time? as it would take me too long now to quote the many results I have. In the meantime, I may say that I have every reason, from my experiments, to take 88 as a fair standard of butter calculation, if associated with at least 6.3 of soluble acids. But I would not apply any charge of admixture to a butter which showed less than 89.5 insoluble with 5 soluble. You will notice that I give the standard of calculation and condemnation differently, and I think this is the proper way, because if a butter really more than passes the utmost possible limit of the article, even when rendered quite unsaleable by decomposition, the admixture being then definitely proved, should be calculated on the fair ordinary standard. This is a point which has been rather lost sight of in milk, and $I$ think we should consider it in fixing any fresh standards. As to any great change in butter by time, calculated to invalidate results of the standards I have given, I believe that when the supporters of that theory get rid of the filter-washing of fats, they will find that apparently enormous changes were due to the fact that now and then by chance they fully estimated their acids. They will also, I think, find, except in the very height of summer, a butter with less than 87 a natural curiosity. It is worthy of note that as soon as admixture steps in, the total acids rise above 95 per cent. I have to thank my chief assistant Mr. De Koningh, an associate of this society, for his accurate work in the practical portion of my researches.

Mr. Otto Hehner said that it was evident that the results obtained by Mr. Angell and himself prior to the publication of their book, were too low in the percentages of fatty acids. Their experience, at that time, led them to assume a standard of $85 \cdot 85$, but they found since that 87 per cent. was the correct proportion. He considered that Dr. Muter's method of obtaining and estimating the volatile acids was a complete confirmation of the process which they had introduced, while, at the same time, he admitted the superior accuracy of Dr. Muter's method of manipulation.

Dr. Dupré took exception to several statements in the paper, and urged the following points :-

Frnst.-That the specific gravity of the melted fat should be compared with water at $60 \mathrm{deg} . \mathrm{F}$, or $62 \mathrm{deg} . \mathrm{F}$, or at $4 \mathrm{deg}$. C.

SECOND.-That the method of heating the fat to the required temperature was not, in his opinion, sufficiently refined. He considered it necessary to keep it at the temperature in a water bath for at least ten minutes before weighing, in order to ensure accurate results.

THIRD.- The thermometer, he considered, should have an elongated bulb, reaching through nearly the entire length of the specific gravity bottle. 
FoбRтн.-That the loss on the basin and beaker used in the experiments which he had made on Hehner and Angell's process, was less than that found by Dr. Muter.

FIFTH.-That the mode of estimating the volatile acids was difficult, and three different estimations entering into the calculation, was more liable to error.

SIxrr. - That the plan described by himself (Dr. Dupré), at the previous meeting, namely heating the butter fat with water in a closed tub to $500 \mathrm{deg}$. F, at which temperature it breaks up into soluble and insoluble fatty acids and glycerine, the first of which can be readily estimated by standard alkali or conversion into barium salt, or secondly by heating butter fat in a closed tub at $500 \mathrm{deg} . \mathrm{F}$ with a standard solution of alkali, afterwards adding a corresponding amount of standard acid, separating the insoluble fatty acids, and estimating the remainder of the acid by deci-normal soda solution, which acid of course corresponds to the soluble acid produced from the butter fat.

Seventr.- That the butter should be melted for some hours before taking the fusing point, and that this should, in crery case, be taken on a rising temperature.

Mr. Wanklyn stated that in his experiments he has found traces only of butyric acid and Dr. Dupre's experiments, which seewed to give different results, showed a loss in the total of more than 4 per cent. He had been led to the conclusion that the amount of butyric acid increased with the age of the butter.

Dr. Dupré pointed out that this loss only occurred in his earlier experiments, when the silver tube leaked, but in his more recent ones, which he should shortly lay before the society, the loss rarely exceeded $\frac{1}{2}$ per cent.

Mr. Allen fully agreed with Dr. Muter's method of stating the specific gravities and also with his directions to dry at $230 \mathrm{deg}$. F., instead of $212 \mathrm{deg}$.

Mr. Turner agreed with Dr. Muter as to the difficulty of washing, and pointed out that the so-called alcohol process which had been associated with his name was really a process which had been applied to the analysis of butter for a number of years past.

Dr. Stevenson said that his experiments led him clearly to the opinion that the fatty acids increased in amount as butter became stale.

After a few other remarks Dr. Muter, in replying, pointed out that he had estimated the free acidity of butter six months old and did not find it exceed 2 per cent., which was within the limits of rariations of samples. He also pointed out two other alternative methods of indirect estimation of the soluble acids. (1) Neutralizing with volumetric potash and then evaporating, igniting and taking the alkalinity of the residue, and (2) a method which he now understood had been previously mentioned by Dr. Dupré, using a standard alcoholic for saponification, and afterwards standard acid for separation of the fatty acids. The objections as yet to these two ways seemed to be (1) the tendency of neutral potassium sulphate to decrepitation and consequent loss, and (2) the difficulty of standard solution in spirit rapidly altering in strength. 